\title{
LE BOEUF DU LAC TCHAD
}

par R. MALBRANT, P. RECEVEUR et R. SABIN, Docteurs vétérinaires.

(3 figures)

Généralement appelé " Kouri " ou "Boudouma ", du nom des groupements indigènes qui en effectuent l'élevage, le bœuf du Lac Tchad constitue, en raison de ses particularités et de ses aptitudes, un dés types de bovidés les plus intéressants du continent africair.

\section{$1{ }^{\circ}$ Origine.}

Historiquement, on ne sait que très peu de choses sur l'origine de cet animal et sur les éleveurs qui le détiennent. Ces derniers seraient, d'après la tradition, des descendants très métissés des Yedina, premiers occupants du Lac, appartenant à un groupement probablement peulh, qui s'est par la suite scindé en deux fractions : celle des Boudoum au Nord 'et celle des Kouri au Sud, ce qui n'apporte d'ailleurs aucune lumière sur l'origine de leur bétail.

Pour Baron, le bœuf du Lac Tchad, considéré par cet auteur comme une race bien définie : “Bos taurus Bolensis " (1), a des grandes ressemblances avec la grande race grise des steppes d'Asie qui, elle aussi, serait d'origine africaine.

Pecaud considère cet animal comme le descendant de taurins peuhl à robe blanche qui se seraient modifiés par l'adaptation à un nouveau genre de vie.

Stewart (2) estime que le bœuf du Lac Tchad est un animal sans bosse, très comparable au bœuf égyptien de l'antiquité dont il serait un descendant à peu près pur.

L'ópinion de Curson (3) est sensiblement différente. Cet auteur, s'inspirant du travail de EPstein (4) considère en effet ce bouf comme un animal à bosse et le range dans la catégorie des pseudo-zébus ou "Sangas ".

(1) Du nom du centre administratif du Lac Tchad qui se trouve à Bol.

(2) Stewart. The Catlle of the Gold Coast. The empire journal of experimental agrieullure. Janvier 1938.

(3) Curson. A contribution to the study of african native cattle. Onderstepoort journal of Veterinary Science. Octobre 1936.

(4) EpsteIn. The origin of Africa's indigenous domestic animals. Cité par Curson comme publis en 1936. 
Des observations que nous avons pu faire, il résulte en effet que le bœuf du Lac Tchad n'est pas un taurin absolument pur et qu'il est légèrement métissé de zébu.

Le travail de Curson, dont nous résumons ci-après quelques-unes des conclusions, fournit de précieux renseignements sur la filiation possible de cet animal. Aussi croyons-nous utile d'en donner un bref aperçu.

Pour eet auteur, le premier bouf domestiqué en Afrique a été le bœuf égyptien «bœuf à grandes cornes » de la valléé du Nil, descendant du Bos primigenius et dénommé par Illzeimer : B. primigenius Hahni. Ce n'est qu'à la fin du néolithique qu'un nouveau type taurin originaire d'Asie, le Bos brachyceros, ful inlroduil sur le continent. Refoulé, d'une part vers le Sud-Est (Haut-Nil, Abyssinie) et, d'autre part, vers le Maroc, en suivant l'Afrique du Nord, puis, de là vers l'Europe par l'Espagne, et vers I'Afrique Occidentale, le Bos primigenius aurait laissé des traces en divers points.

L'arrivée du Bos brachyceros dans le bassin inférieur du Nil fut suivie de près par l'introduction des premiers zébus asiatiques, à bosse musculaire située en position cervico-dorsale, dénommés « zébu à cornes latérales » par Epstein; Curson et Thornton, dont les détenteurs refoulèrent à leur tour les derniers arrivés vers l'Uuest et vers le Sud. Il s'en suivit évidemment de nombreux métissages et les seuls "zébus à cornes latérales " qui purent en être préservés furent ceux appartenant au peuple Hottentot actuel que ses migrations poussèrent jusqu'en Afrique du Sud, où il se trouve encore aujourd'hui, et qui, isolé de tout autre groupement d'éleveurs; conserva cette race bovine à l'état pur (race Afrikander).

Dans le haut bassin du Nil, c'est le croisement du zébu à cornes latérales avec le Bos primigenius précédemment refoulé qui fournit le bovidé dénommé “ Sanga ", type stable et à caractères non reverșibles, dont le bœuf du Lac Tchad ne serait qu'un descendant. En effet, d'après Curson, au début de l'ère chrétienne, les tribus Bantous, v'enues de l'Abyssinie et Haut Nil y importèrent cette race en passant par le Soudan Anglo-Egyptien et le Nord de la zone à tsé-tsé de l'A.E.F., tandis qu'une autre fraction de même origine émigrait vers l'Ougánda et se dispersait de là en diverses régions de l'hémisphère austral (Congo Belge et territoires voisins du Sud-Est et du Sud-Ouest Africain).

A peu près à la même époque (entre 100 et 500 après J.-C.) arrivait et se dispersait en Afrique une autre souche de bovins : le zébu à courtes cornes; du type Bos'indicus, caractérisé par une 'bosse musculo-adipeuse bien développée et située en position dorsale. C'ést le croisement de ce zébu avec le bœuf égyptien, ou ses descendants, qui aurait fourni le zébu à cornes en lyre, aujourd'hui commun dans l'Ouest Africain. 


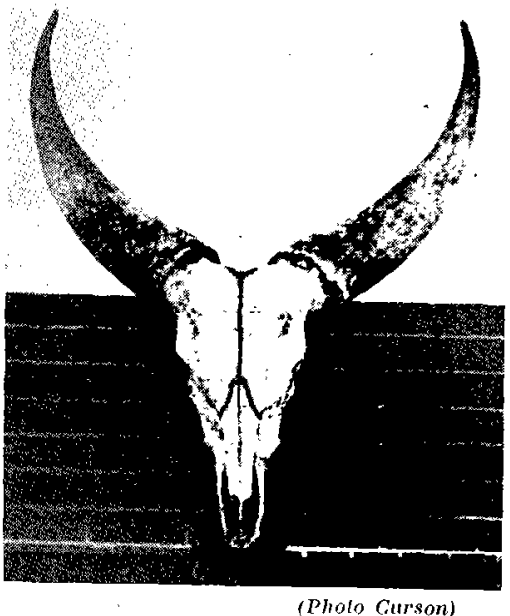

Fig. 1.- Crâne de bœuf du Lac Tehad.

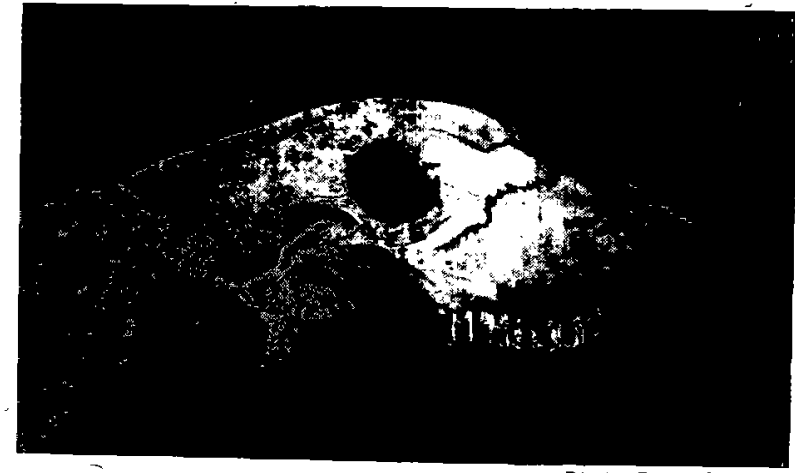

Fig. 2. - Crâne de bœuf du Lae Tchad.

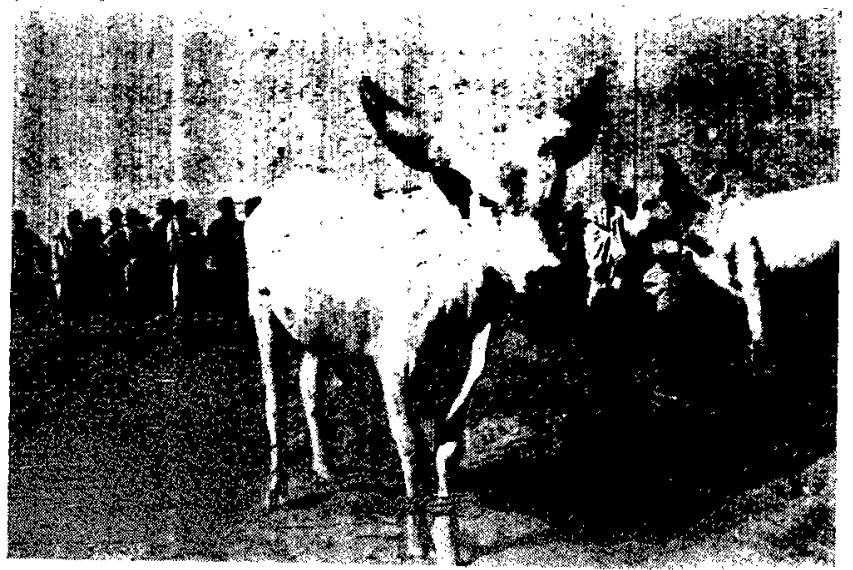

Fig. 3. - Taurillon Kouri. 
D'après la théorie qui précède, les bovidés africains se classeraient done ainsi qu'il suit :

$1^{\circ}$ Bovipés sans Bosst..... $\left\{\begin{array}{c}\text { a) Bos primigenius (dont le bœuf N'Dama et le } \\ \text { bœuf des Mandingues du Libéria seraient } \\ \text { des descendants peu modifiés). } \\ \text { b) Bos brachyceros (bœuf des Lagunes du } \\ \text { Dahomey, etc...). }\end{array}\right.$

a) Zébu à cornes latérales, caractérisé par une bosse muscülaire bien développée et située en position cèrvico-dorsale (Zébu Afrikander).

b) Pseudo-zébu Sanga, produit de croisement dù B. primigenius et du zébu à cornes latérales (bouf du Lac Tchad et nombreux pseudo-zébus du Centre, de l'Est et du Sud africain).

c) Zébu d̀ courtes cornes, descendant du Bosindicus, caractérisé par unè bosse musculo adipeuse, généralement très développée et située en position dorsale (nombreuses races africaines).

d) Zébu d̀ cornes en lyre, produit du croisement du zébu à courtes' cornes' avec le Bos primigenius ou le Sanga (1). Ce zébu serait caractérisé par son cornage en lyre, et par une bosse musculo-adipeuse bien développée et située en position cervico-dorsale. C'est à ce type qu'appartiendrait le zébu' Bororo et plusieurs races de zébus peulh de l'Duest africain.

Chronologiquement, ces introductions et ces migrations se seraient effectuées de la façon suivante :

Domestication par les Egyptiens du boeuf sauvage à grandes cornes (Bos primigenius Hahni) ... . . ..................

Introduction en Afrique du Bos brachyceros ...

Emigration vers lOuest africain du $B$. primi-

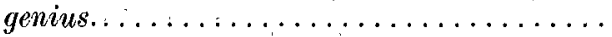

Introduction en Afrique du zébu à cornes laté-

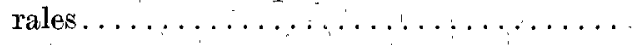

Constitution de la race Sanga (pseudo-zébu obtenu pai le croisement de Bos primigenius. et du zébu à cornes latérales).......... aủ début de l'ère chrétienne.

(1) Après ávoir adop̣tá cette thèse, STrwakr n'est toutefois plus de cet avis et il tend à considérer le zébu à cornes en lỳre comme un descendant du zébu d'Asie (Lettre personnelle, mai 1941). 
Dispersion de cette race en diverses régions d'Afrique

premiers siècles de l'ère chrétienne.

Introduction et dispersion en Afrique du zébu à courtes cornes ....................

Constitution de la race zébu à cornes en lyre .. temps historiques.

En ce qui concerne plus spécialement le "Sanga ", il serait, d'après Curson, ainsi caractérisé : Front large. Profil généralement rectiligne. Arcades orbitaires proéminentes. Apophyse épineuse des vertèbres dorsales simple ou bifide à son extrémité. Bosse musculaire peu développée, située en position cervico-dorsale, c'est-à-dire à cheval sur les dernières vertèbres cervicales et les premières dorsales, alors que la bosse de certains zébus, notamment ceux à cornes en lyre (zébu foulbé par exemple) est musculo-adipeuse et située en position plus postérieure (premières vertèbres dorsales). Ses autres particularités seraient intermédiaires entre celles du bœuf égyptien et du zébu à cornes latérales.

Le bœuf du Tchad s'écarte un peu du type ainsi défini. Car s'il est exact que, même dans les îles du Lac où il s'est le mieux trouvé préservé des métissages, cet animal présente toujours au moins un rudiment de bosse musculaire situé en position cervico-dorsale, il n'en est pas moins certain que ses affinités générales sont beaucoup plus proches du type taurin que du type zébu. Il ne peut donc ètre classé parmi les bovins du type Sanga chez lesquels la proportion de sang zébu et de sang taurin est à peu près de même importance et il convient à notre avis de le considérer comme un taurin légèrement métissé soit de Sanga soit de zébu à cornes latérales (1).

\section{Habitat.}

Le bœuf Kouri ne se rencontre dans son type vraiment pur que dans la région insulaire du Lac Tchad. C'est dans la région des îles de Djibadala, Koremerom, Debada, Bagabol, que semblent se trouver les spécimens les plus représentatifs de la race. Sur le pourtour, notamment dans la zone habitée à l'Est par les Kanembous, cet animal a donné naissance, par croisement avec des zébus vrais, à de nombreux métis dont le degré de sang taurin diminue graduellement à mesure que l'on s'éloigne du Lac.

D'après Chudeau, le bœuf du Lac Tchad ne se retrouverait en dehors de cette zone que chez des Toubous établis au Nord du Koutous (300 km. à l'Ouest du Lac) et chez les Motbeurs, tribu d'origine Kanembou et Sô du district de Bakara (Bornou). Ces renseignements

(1) Il est à noter cependant que, dans le Nord du Lac Tehad (Région de N' Guimni) le bœuf Kouri semble s'être conservé beaucoup plus pur et que l'influence zébu y apparaît généralement moins marquée. 
nous ont été confirmés par un méhariste européen, M. Viguier, qui a longtemps vécu dans ces régions. Les animaux existant, plus ou moins métissés de zébu, sont entre les mains des Toubous, qui fréquentent le puits de Tass et des Dagras Coutis de la région du Manga (Cercle de Gouré) et qui se déplacent entre les puits de Tass où ils sont en contact avec les Toubous, Biltoum, Bouloum et Kalle.

Quant aux Motbeurs, ils sont relativement très voisins du Lac, occupant les rives de la Komadougou, dans le cercle de N'Guigmi. Au cours de ces dèrnières années, des essais d'introduction de cette race ont eu lieu en diverses régions, soit en vue d'en pratiquer l'élevage à l'état pur, soit en vue de métissage. Cela a notamment été le cas :

Au Tchad, au Lac Fittri, au Bas-Chari et à la Station agronomique du Tickem (Lac de Fianga en région soudanaise). Les expériences, faites sur une trop petite échelle et surtout insuffisamment. suivies sont à reprendre. A noter cependant que les bœufs de la ferme du Tickem se sont correctement entretenus;

au Niger dans la région de Tillabery;

en Nigéria, à Maïduguri, importation par les Anglais en 1944 d'un noyau de reproducteurs composé de 10 vaches et d'un taureau.

(A suivre.) 